\title{
3D Computation of Broadband Slat Noise from Swept and Unswept High-Lift Wing Sections
}

\author{
J. Dierke, C. Appel, J. Siebert, M. Bauer*, M. Siefert*and R. Ewert* \\ Institute of Aerodynamics and Flow Technology, Technical Acoustics \\ German Aerospace Center (DLR), \\ Lilienthalplatz 7, 381208 Braunschweig, Germany
}

\begin{abstract}
In previous work a RANS based simulation technique for the simulation of broadband slat noise was established. Good agreement was found between predicted and measured slat noise spectra. These predictions were based on $2 \mathrm{D}$ CAA computations and a connection to 3D measured data is only possible assuming a certain functional behavior of the spanwise coherence of the essential slat noise source. For this purpose, results from trailing edge noise measurements were used. In this work the simulation strategy is extended to 3D CAA computations, resolving the spanwise slat noise coherence as part of the CAA computations. The considered wing span is one main-chord, which is large enough to establish a realistic 3D problem for the turbulence as well as for the sound radiation. The Fast Random Particle Mesh (FRPM) method is applied for this study to generate fluctuating sound sources from steady RANS turbulence statistics. The study is conducted for the 30P30N airfoil with $0.457 \mathrm{~m}$ main chord. The Mach number is 0.17 and the angle of attack is $4^{\circ}$. Good agreement is found between the previous $2 \mathrm{D}$ and the $3 \mathrm{D}$ results as well as with unsteady simulations published in the literature. The influence of sweep on slat noise generation is studied.
\end{abstract}

\section{Introduction}

Developing quieter aircraft is still considered an important factor to enable the foreseen growth of air traffic in the future. One of the main sound sources during approach is airframe noise. To be able to develop quieter aircraft the understanding of sound sources is helpful and an efficient and fast computation capability for airframe noise problems mandatory. The fast simulation of broadband noise depends on the rapid provision of unsteady turbulent sound sources.

The Random Particle Mesh (RPM) method ${ }^{1,2}$ is a synthetic turbulence generator that enables the generation of fluctuating sound sources from RANS, taking into account the major specifications that have to be realized to be appropriate for an application to aeroacoustic problems. The sound propagation in a viscous mean flow is computed with the Computational Aero Acoustics (CAA) code PIANO, developed at DLR.

Considerable work has been spent in recent years on the numerical and analytical prediction of slat noise. Agarval and Morris ${ }^{3}$ presented a numerical prediction method for slat noise which is based on a 2D Boundary Element Method (BEM) for uniform flow in conjunction with a statistical noise source model akin to the jet noise source proposed by Tam \& Auriault. ${ }^{4}$ However they give no numerical prove whether their model provides the proper Mach number scaling law for slat noise. Pott-Pollenske et al. proposed a semiempirical prediction tool for airframe noise including slats. Koenig et. al. identified the perturbed Lamb vector as a major acoustic source in the slat region based on LES computation ${ }^{5}$ with a spanwise dimension of $2 \%$ chord length $(c)$. Ma and Zhang used LES to get the acoustic sources in combination with acoustic perturbation equations (APE) for the wave propagation. They investigated the potential for noise control using acoustic liners in the slat region. ${ }^{6}$ Imamura et al. computed the unsteady flow around a conventional slat using a zonal-LES/RANS code. ${ }^{7}$ Their focus was on the near field flow structures. Lockard and Choudhari performed hybrid RANS/LES computations for the 30P30N geometry. ${ }^{8,9}$ They studied the flow

*Research Scientist, Technical Acoustics, email:juergen.dierke@dlr.de, and AIAA Member 
near the flap for different sweep angles and provided far field spectra via FW-H extrapolation. The spanwise extension varied from $5.6 \%$ to $33.9 \%$ c. The cross flow seems not to have a significant influence on the radiated noise. Guo presented a model for aircraft slat noise predictions based on the theory of aerodynamic sound generation, taking into account various mechanisms for noise generation and propagation. ${ }^{10}$

It has been shown in the EU projects TIMPAN ${ }^{2}$ and LEISA that the RPM method (Random Particle Mesh) yields very good aeroacoustic predictions. But so far these predictions were based only on 2D computations. This paper will show, that the new improved version, the FRPM (Fast Random Particle Mesh) ${ }^{1}$ method, developed by Ewert and Siefert, produces results as good as RPM and also covers 3D problems. The extension of $2 \mathrm{D}$ calculations to $3 \mathrm{D}$ assume a frequency dependent coherence length scale in spanwise direction, which was inferred from the surface pressure characteristics at a trailing edge. ${ }^{11}$ A $3 \mathrm{D}$ calculations model provides the whole stochastic field and therefore includes the spanwise source statistics. The advantage of the RPM and FRPM methods in contrast to LES, DES etc. is the possibility to place turbulent sound sources at various locations, which enables to study their acoustic effects and influence separately. Yet another advantage is the significantly reduced computational time. A 2D FRPM computation for a slat noise prediction for $0.14 s$ takes around $1 d$ on 8 CPUs. The cluster consists out of Intel Xeon $2.93 G H z$ processors.

The 30P30N geometry is used in this paper. It consists of a slat, wing and flap in landing positions. The high lift airfoil is used in two ways, first unswept to enable a comparison of RPM and FRPM results as well as a comparison with 3D FRPM results. The spanwise direction is resolved for one chord length, which allows for the development of a real 3D sound field. Then in a second step the airfoil is used with a sweep angle of $30^{\circ}$ in such a way, that the flow properties in a plane normal to the leading edge are the same as for the unswept wing. The results are compared against results computed with DLR's DG-CAA code and against the generic spectra proposed by Guo. ${ }^{10}$

\section{Aeroacoustic Simulation Approach}

Acoustic Perturbation Equations (APE) are the governing equations applied for the computations. The CAA Code PIANO ${ }^{12}$ applies curvilinear structured grids. Spatial gradients are approximated by using the dispersion relation preserving (DRP) scheme proposed by Tam and Webb. ${ }^{13}$ The temporal discretization is achieved with the 4th-order lowdispersion Runge-Kutta (LDDRK) algorithm proposed by Hu. ${ }^{14}$ As a mean-flow a RANS solution was computed for a high-lift geometry with landing settings, using the unstructured flow solver TAU of DLR. The sound sources are modeled by the RPM and FRPM method.

\section{II.A. Mathematical Framework of (F)RPM}

The (F)RPM framework was was introduced in Refs. ${ }^{15-18}$ as a stochastic method to generate synthetic turbulence with locally prescribed one- and two-point statistics, with special focus of its appli-

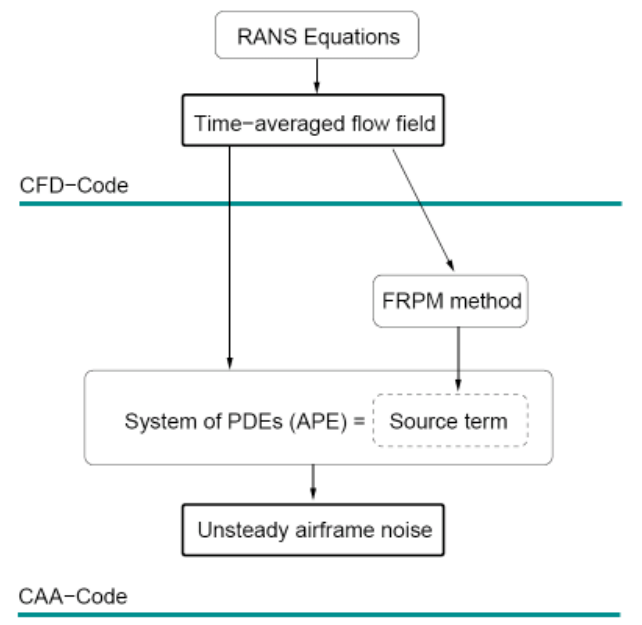
cability in CAA. It provides in an Eulerian frame a statistically stationary fluctuating sound source, which combines source convection with temporal decorrelation models. Due to the inter-relation of temporal and spatial properties the method is considered a 4-D synthetic turbulence model.

Fig. 2 gives a general overview of its theoretical background. The theoretical framework (Fig. 1) allows to derived the realized cross-correlations (left branch in Fig. 2). The theoretical framework rests upon the novel concept of convective white noise. A consistent numerical discretization leads to a numerical realization with random particles (right branch in Fig. 2). Hence, within the limits of accuracy of the numerical approach the particle model provides fluctuations which possess the derived cross-correlations (horizontal arrow in Fig. 2).

Two different numerical procedures have been proposed to realize the particle model, namely RPM and 


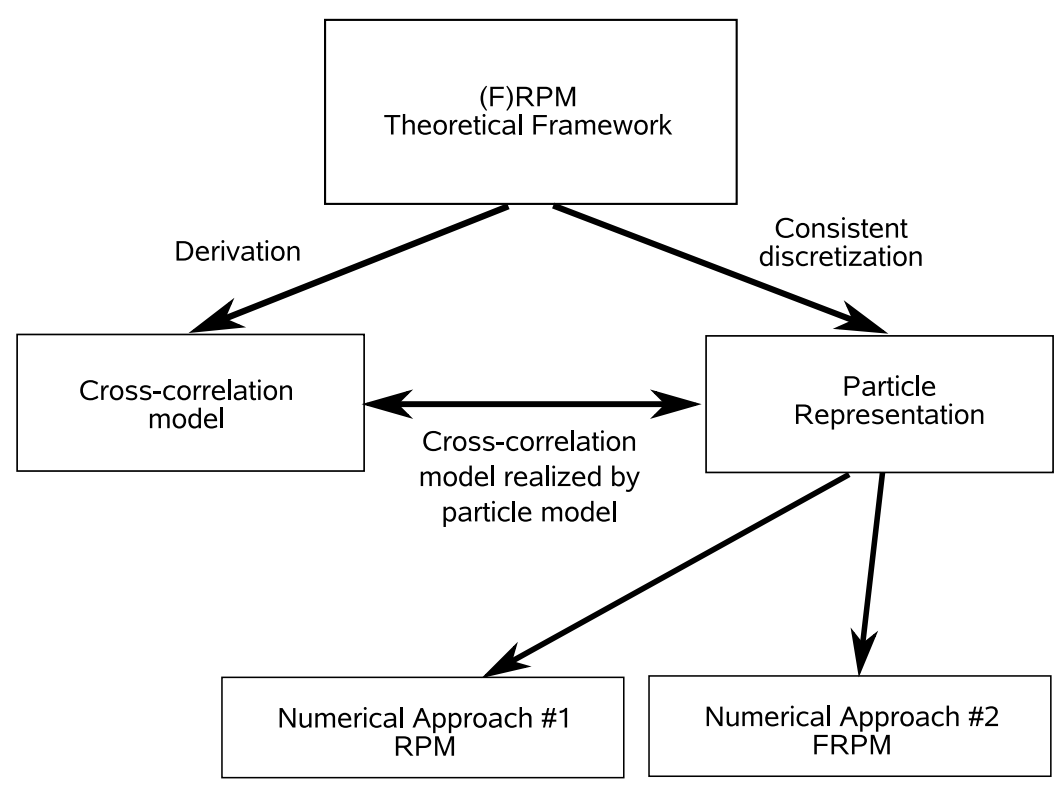

Figure 2. Overview over the theoretical background of the Random Particle Mesh method.

FRPM. Both approaches have in common that convecting particles are used, which carry random values.

To each particle a set of mutually uncorrelated random variables $r_{i k}$ is assigned. Their amount defines the number of fluctuating source components generated. The first index $i$ denotes the component of the random variable set, the second index indicates the considered particle. For spatial 2-D problems one fluctuating source component is realized, while in 3-D there are three independent source components used.

A specific fluctuating source component $\psi_{i}(\boldsymbol{x}, t)$ is generated through the weighted sum over all $N$ particles, summing up the random variates associated to the source component $i$, i.e.

$$
\psi_{i}(\boldsymbol{x}, t) \simeq \hat{A}(\boldsymbol{x}) \sum_{k=1}^{N} G\left(\boldsymbol{x}-\boldsymbol{x}_{k}^{c}(t)\right) \frac{r_{i k}(t)}{\rho_{0}\left(\boldsymbol{x}_{k}^{c}\right)} .
$$

The function $\hat{A}$ specifies the variance of the fluctuations realized. It can be a given as a function of either in particle or mesh coordinates, i.e. $\hat{A}\left(\boldsymbol{x}^{\prime}\right)$ or $\hat{A}(\boldsymbol{x})$, respectively. In this work a Gaussian filter kernel is applied for $G$. Each particle represents the aforementioned convective white-noise field over a representative control volume surrounding each particle. The random variables satisfy the conditions

$$
\begin{aligned}
\left\langle r_{i k}(t)\right\rangle & =0 \\
\left\langle r_{i k}(t) r_{j l}(t)\right\rangle & =\delta_{i j} \delta_{k l} \delta m_{k} \\
\dot{r}_{i k} & =-\frac{1}{\tau_{s}} r_{i k}+\sqrt{\frac{2}{\tau_{s}}} s_{i k} \dot{r}_{i k} \\
\dot{\boldsymbol{x}}_{k}^{c} & =\boldsymbol{u}_{0}\left(\boldsymbol{x}_{k}^{c}\right) .
\end{aligned}
$$

In plain words, the random variables $r_{i k}$ are mutually uncorrelated with vanishing mean and a constant variance proportional to $\delta m_{k}$, which is the fluid mass encompassed by the control volume surrounding each particle. The particle drifts with the local mean flow velocity at the particle position $\boldsymbol{x}_{k}^{c}$. The random values are time independent for frozen turbulence $\left(\tau_{s} \rightarrow \infty\right)$, otherwise exhibit an exponential time decorrelation.

\section{II.A.1. FRPM Discretization}

The Fast RPM (FRPM) approach makes use of a Cartesian auxiliary mesh, on which random particles freely float, Fig. 3. A cloud of $N$ particles evenly covers the Cartesian background mesh. The source amplitude $\hat{A}$, as well as length and time scales together with the mean flow and the mean-flow density are made available 
on the auxiliary mesh by linearly interpolating the values from the vertices of the cell hosting the particle to the particle position itself. The synthetic fluctuating sound sources are computed in the cell vertices of the auxiliary mesh.

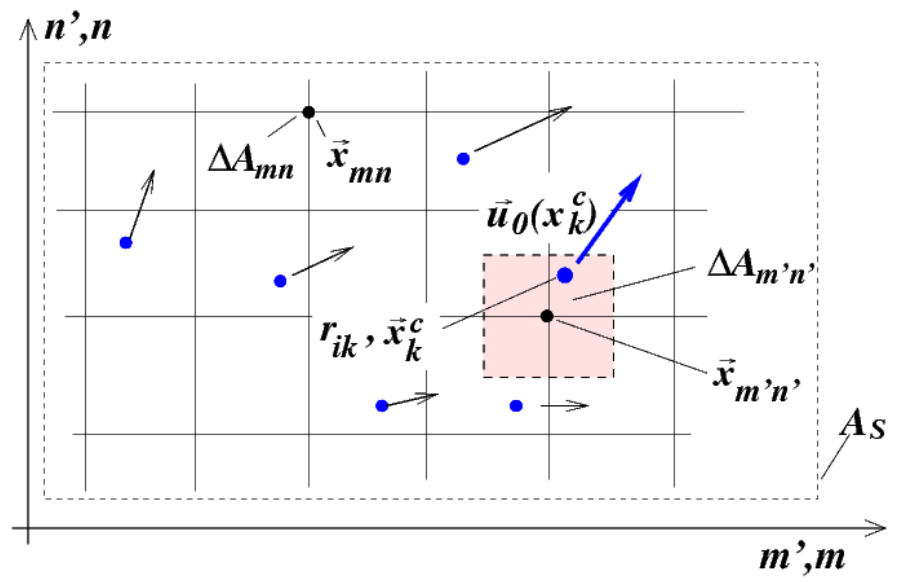

Figure 3. Random particles and notations for a 2-D auxiliary FRPM mesh; random particles and notations for a 2-D auxiliary FRPM mesh, $\Delta A_{m^{\prime} n^{\prime}}$ denotes the node centered control volume around vertex $\left(m^{\prime}, n^{\prime}\right)$.

The FRPM discretization realizes white-noise up to a wave-number cut-off defined by the spatial resolution of the auxiliary mesh. Compared to the initial RPM discretization, the FRPM approach mainly has the advantage that more easily recirculation areas in the mean-flow fields can be taken into account. Furthermore, in the FRPM approach very efficient Gaussian filters based on recursive filters can be used, ${ }^{19}$ which allow to reduce the filtering effort by more than one order of magnitude (compared to a naive application of sequential 1-D Gaussian filtering). The speed-up becomes even more significant for spatial 3-D applications.

\section{II.A.2. Sound source}

For the simulation of airframe noise we apply Acoustic Perturbation Equations (APE-4) with sources (for their formulation refer e.g. $\mathrm{to}^{20}$ ) as the acoustic propagation model. The homogeneous equation system is equivalent to the convective wave equation of irrotational flow. In previous studies a vortex force term on the right-hand side of the momentum equation could be identified as the most important source term. The source term reads

$$
\overrightarrow{q_{m}}=-\underbrace{\vec{\omega}_{0} \times \vec{u}^{\prime}}_{I}-\underbrace{\vec{\omega}^{\prime} \times \vec{u}_{0}}_{I I}-\underbrace{\left(\vec{\omega}^{\prime} \times \vec{u}^{\prime}\right)}_{I I I}
$$

in which $\omega$ and $u$ are the vorticity and velocity respectively, while an index zero denotes a mean flow quantity and a prime denotes a(turbulent) perturbation about this mean. The non-linear term III can be considered smaller than the linear terms $I$ and $I I$. The source term Eq. 6 is in general composed out of a rotational part defined by $\nabla \times \vec{q}_{m} \neq 0$, and an irrotational part defined by $\nabla \cdot \vec{q}_{m} \neq 0$. Direct turbulent sources can be attributed to their irrotational part whereas the rotational part primarily is responsible for the generation of vorticity in the APE, which can be seen by taking the curl of the APE momentum equation. Altogether the source term yields

$$
\frac{\partial \omega^{\prime}}{\partial t}=\nabla \times \overrightarrow{q_{m}}
$$

This shows that the induced vorticity on the left-hand side is entirely controlled by the rotational part of the momentum source. The approach has the advantage that the actual dynamics of the induced vorticity very closely follows the dynamics as prescribed by the source. With a propagation model based on e.g. the linearized Euler equations (LEE) this is not necessarily the case, since partly vortex dynamics are directly resolved by the propagation equations and partly stimulated by the source. It may occur that the effectively induced vorticity dynamics not exactly follows the behaviour as expected to be induced by the source term. One essential difference that appears in comparison with traditional acoustic analogy that the sources not only act as an acoustic source, but rather induces vorticity as well. Sound is generated as part of the acoustic simulation process due to the interaction of the airframe with the vorticity stimulated by the source. This is e.g. different to other statistical airframe noise approaches which rely on the availability of a surface pressure 
spectrum. A vorticity based source model gives a more direct connection with turbulence properties and the generated sound. It is well known that free turbulence generates a sound intensity following an eight power law, whereas airframe sound generation scales with a somewhat smaller, i.e., fifth power law. Hence, for small Mach numbers, the airframe noise mechanism is clearly dominating over the sound generation process of free turbulence. This means that for airframe noise simulation the rotational part of the momentum source term is the most important contribution.

\section{Source term B}

The vorticity in term II can be modeled directly by (F)RPM. In 2D a scalar quantity generated by (F)RPM can be interpreted as the third component of fluctuating vorticity $\left(\omega_{3}^{\prime}\right)$. In $3 \mathrm{D}$ a vector with three independent realisations is generated and interpreted as $\vec{\omega}^{\prime}$. Often it is assumed that the vorticity components are uncorrelated to the velocity fluctuations, i.e. velocity could be generated by anadditional mutally un-correlated (F)RPM realization. Here however, we assume that the contribution of term $I$ has less importance than term $I I$ and model only term $I$, i.e. $q_{m}=-\vec{\omega}^{\prime} \times \overrightarrow{u_{0}}$, which we will subsequently call "source B". For the $2 \mathrm{D}$ source formulation $\mathrm{B}$ the variance of the fluctuating quantity provided by (F)RPM has to be matched to the vorticity variance. To achieve the right vorticity levels the vorticity variance must scale according to

$$
\left\langle\omega^{\prime 2}\right\rangle \propto \frac{k}{l_{s}^{2}} \propto \omega^{2}
$$

where $l_{s}$ and $k$ are the length scale and the turbulence kinetic energy from RANS, respectively, and $\omega$ denotes the specific dissipation rate. The integral length scale of vorticity is also matched to the length scale $l_{s}$. Note, in principle the integral length scale should be proportional to the Taylor length scale, which has for high Reynolds numbers an intermediate size between the Kolmogorov length scale and integral length scale $l_{s}$. However, the (F)RPM generated spectra can be deemed to represent a truncated part of the full turbulent spectrum, i.e. including the sound generated by the most energetic part of the turbulent spectrum by omitting high-frequency contribution of smaller turbulence. Hence, the generated Gaussian spectrum could be seen as a model to describe a truncated full turbulent spectrum. Consequently, the vorticity spectrum derives only from resolved velocity components, thus exhibiting a larger length scale proportional to $l_{s}$.

\section{Set-up}

The numerical set-up is a 30P30N high-lift profile at landing configuration with a clean chord length of $0.457 \mathrm{~m}$ and a free stream Mach number of 0.17 , which leads to a Reynolds number of 1.7 million. The angle of attack is $4^{\circ}$. The Mach number and angle of attack for the swept case are listed in Table 1 below. The angle of attack and Mach number normal to the leading edge are kept constant at 0.17 and $4^{\circ}$ for all cases.

\begin{tabular}{|c|c|c|c|}
\hline AoA & Mach number & Sweep angle $\beta$ & Reynolds number \\
\hline $4^{\circ}$ & 0.17 & $0^{\circ}$ & $1.7 E 6$ \\
$3.4655^{\circ}$ & 0.196 & $30^{\circ}$ & $1.7 E 6$ \\
$4^{\circ}$ & 0.17 & $0^{\circ}$ & $10 . E 6$ \\
\hline
\end{tabular}

Table 1. Table of flow parameters

The RANS calculations were performed using the DLR TAU code. Two different grids were built, one $2 \mathrm{D}$ mesh and one 3D mesh for the swept wing. The numerical $2 \mathrm{D}$ grid is composed of 380000 nodes. The boundary layers are resolved by 50 nodes with the first interior point located at $y^{+} \leq 1$. Farfield boundaries are located at $75 l_{c}$ distance from the airfoil. The $3 \mathrm{D}$ grid consists of $8 . E 6$ points. The boundary layers are resolved by 30 nodes with a $y^{+} \leq 1$. The farfield boundaries are located $5 l_{c}$ away form the airfoil. Periodic boundaries are utilized for the span-wise boundaries.

The RANS computations were conducted using a Menter SST turbulence model.The basic equations are discretized with a central difference scheme and the turbulence equations by a 2nd order accurate upwind scheme, which is necessary to yield a high resolution of both the specific dissipation rate and the turbulence length scale.

The 2D CAA mesh is designed to resolve frequencies up $15 \mathrm{kHz}$ in the area beneath the profile with 7 points per wavelength. It comprises 49 blocks and around $370 K$ points. The point density is increased in

5 of 13

American Institute of Aeronautics and Astronautics 
the source area to resolve the turbulent vortices. The mesh is smoothed across the block boundaries to get the best possible results. The 3D mesh is derived from this $2 \mathrm{D}$ mesh by extruding in spanwise direction. A wing span of to 1 full chord length is realized. The mesh is stretched in such a way that it resolves the acoustic wave length in the outer region and the turbulence in the inner region. The source area's dimension is plotted in Fig. 14 .

\section{Results}

The results are sorted in three categories. In the first section the differences in pressure distributions and turbulence topologies of the RANS simulations are discussed. The second subsection presents the source statistics. And finally the acoustic results are presented. The flow coordinate systems are sketched in Fig. 16.

\section{IV.A. RANS Results}
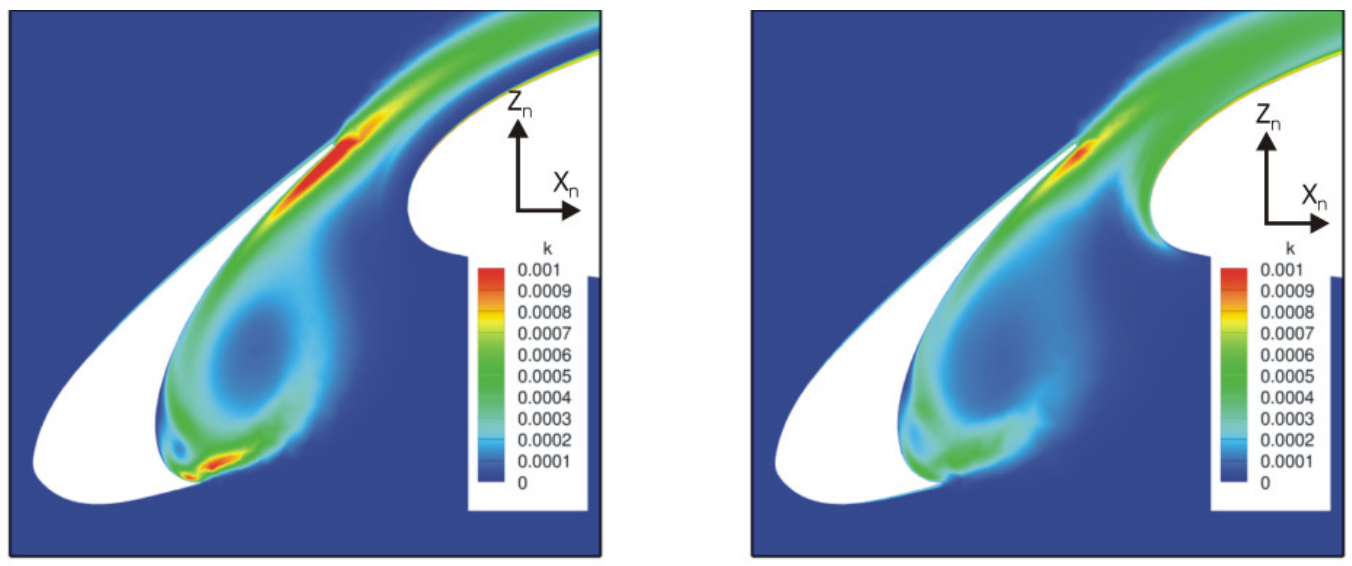

Figure 4. Turbulent energy $(k)$ distribution on slices in the $x_{n}-z_{n}$-plane; left for the unswept case and to the right for the swept case.

The RANS results for the swept and unswept wing show similar topologies for the turbulent kinetic energy distribution in the slat region (Fig. 4). The kinetic energy near the nose of the swept wing is probably caused due to the coarser 3D mesh. The amplitude " $k$ " at the lower side of the slat trailing edge is slightly lower for the swept wing.
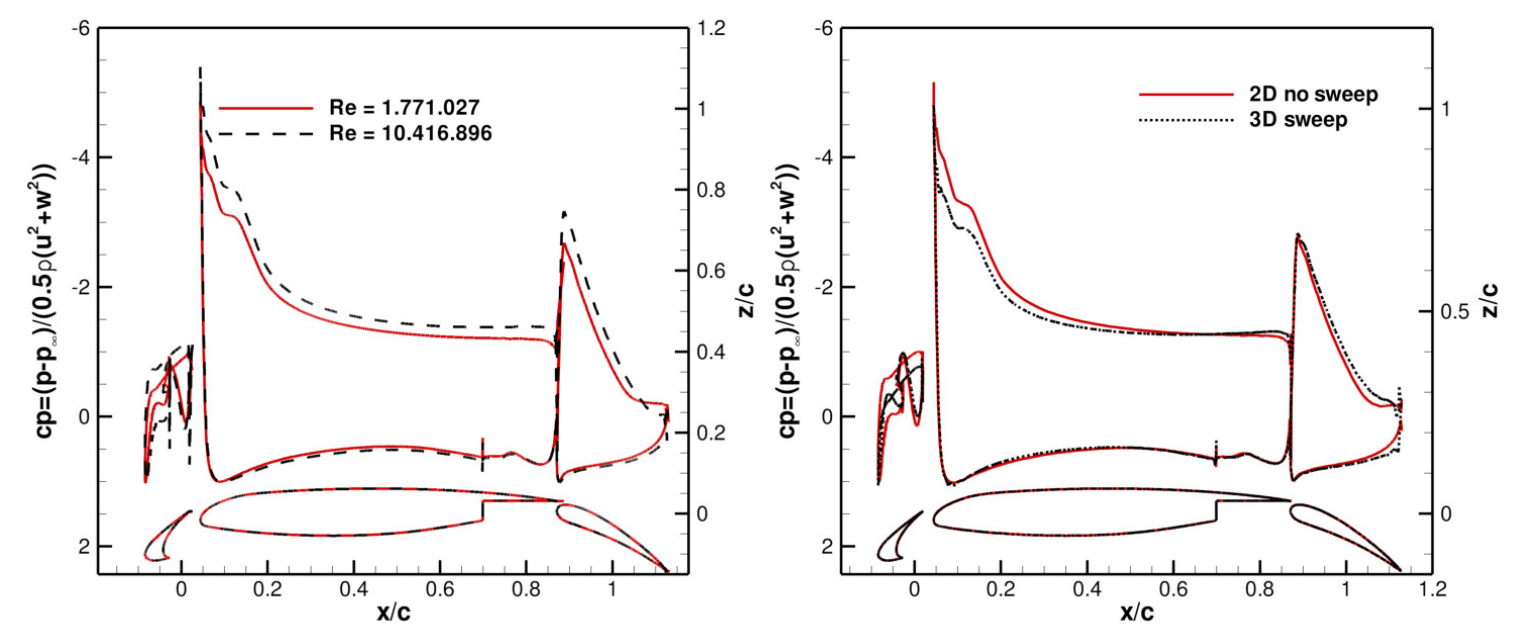

Figure 5. Pressure coefficient distrubutions; at the left for the unswept cases for Reynolds numbers 1.7 and 10 million; to the right the $c_{p}$ distributions for the swept and unswept wing

In Fig. 5 the pressure coefficient $c_{p}$ of the wing without sweep is shown for two different Reynolds numbers. 
The $c_{p}$-distributions have a similar shape but with higher load for the higher Reynolds number (Fig. 5(a)). Only the flap trailing edge shows smaller $c_{p}$ values for the high Reynolds number case.

Fig. 5(b) depicts the $c_{p}$-distribution of the swept and unswept wing. The pressure coefficient is definied as $c_{p}=\left(p-p_{\infty}\right) / \frac{\rho}{2}\left(u_{n}^{2}+w_{n}^{2}\right)$. The distributions are quite similar. Differences are observed at the slat and wing leading edge, where the sweep causes lower $c_{p}$ values. At the flap trailing edge however, sweep causes higher $c_{p}$ values.

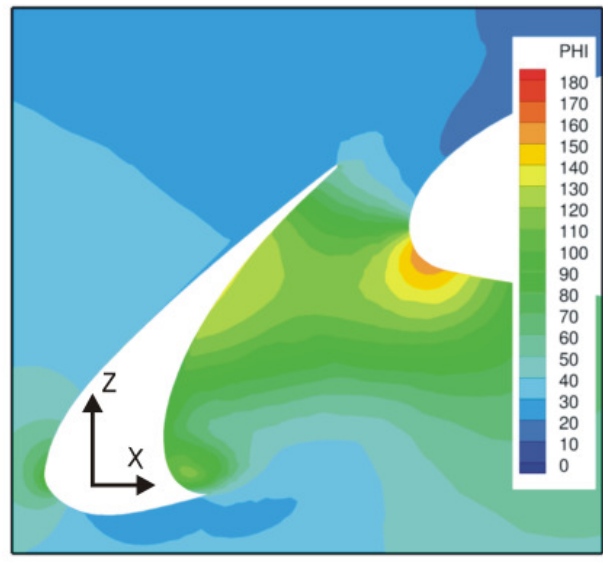

(a)

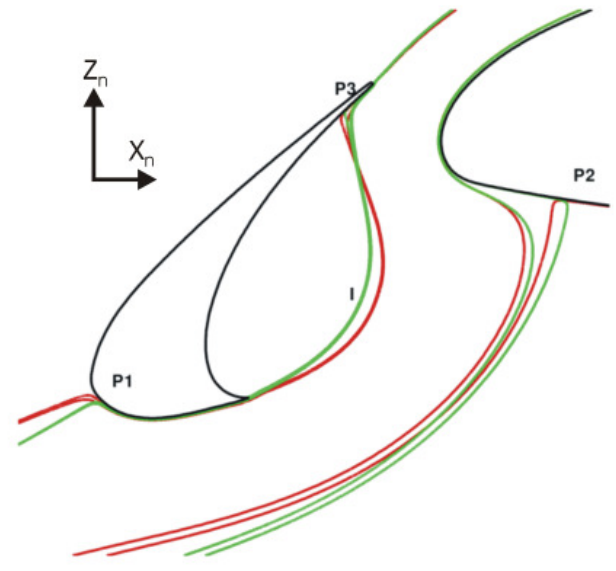

(b)

Figure 6. Angle PHI between $x$ and the velocity vector(a); Stream traces for the swept (red) and unswept (green) wing near the slat trailing edge (b)

The mean flow is influenced by the sweep angle especially in the slat region. Figure 6(a) shows inclination angles "PHI" in the slat cove, which is defined as the angle between $\mathrm{x}$ and velocity. This angle should correspond to sideslip $\beta$ in the far field, but at the slat it exhibits a more complex behaviour. Below the slat hook the direction is around $30^{\circ}$, then, along the shear layer that separates the slat cove recirculation curl from the gap flow, it increases to a flow parallel to the wing leading edge. Finally it decreases again to angles slightly higher than $30^{\circ}$ near the slat trailing edge and it is again $30^{\circ}$ behind the slat trailing edge. The mean flow lies nearly in the plane normal to the wing leading edge at the slat trailing edge. The stream traces in the normal plane are depicted in 6 . The separation line of the unswept wing hits the slat slightly behind the line of the swept one. The stagnation point stream traces hitting the slat and main element are slightly shifted to the front of the geometry in case of sweep.

\section{IV.B. Source statistics}

Here, the statistics of the modeled source is presented. A considerable number of sensors are put into the source area to enable the calculation of the modeled correlation length scales and convection velocity (Fig. 15). Sensors 1-50 are located in spanwise direction in the area of the maximum of turbulence energy near the slat trailing edge. Microphones 51-80 follow a stream trace for the unswept case crossing the maximum again. Sensors 81-130 follow a swept stream trace.

As expected, the spanwise correlation has the shape of a Gaussian (Fig. 7). The integral length scale $l$ follows from the integral of the correlation function over the distance $l=\int_{0}^{\infty} R(r) d r$. The Gaussian has the shape $R=\exp \left(-\frac{\pi}{4} \frac{(\Delta r)^{2}}{l^{2}}\right)$. The shape fits quite well and the target length scale of 0.004 is nearly reached by the integral length scale with 0.0039 .

Fig. 7b shows the decorrelation along a stream trace. Evolving turbulence is described by the model even if no explicit time-decorrelation model used (e.g. based on a Langevin equation). Here the decorrelation is merely caused by the non-uniform convection realized for the synthetic turbulence. The theoretical shape of an envelope for shear decorrelation follows $R=1 / \sqrt{1+\alpha^{2} \tau^{2}}$. The coefficient $\alpha$ can be derived from RANS data by: $\alpha=\frac{1}{2}\left(\frac{d u}{d y}\right)^{2}$. The envelope describes the temporal decrease of the correlation coefficient very well. 


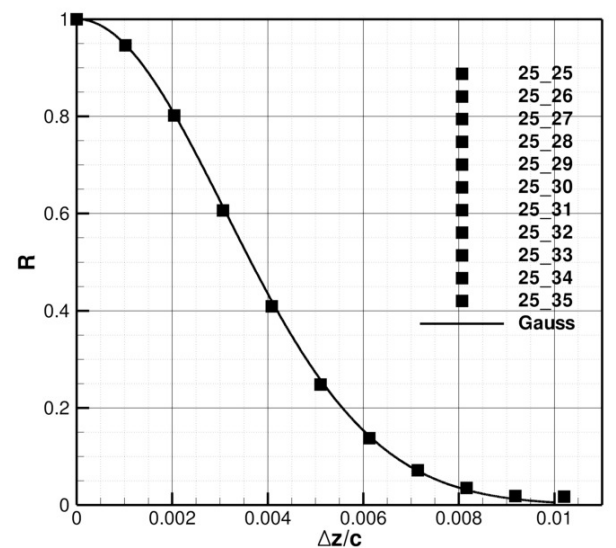

(a)

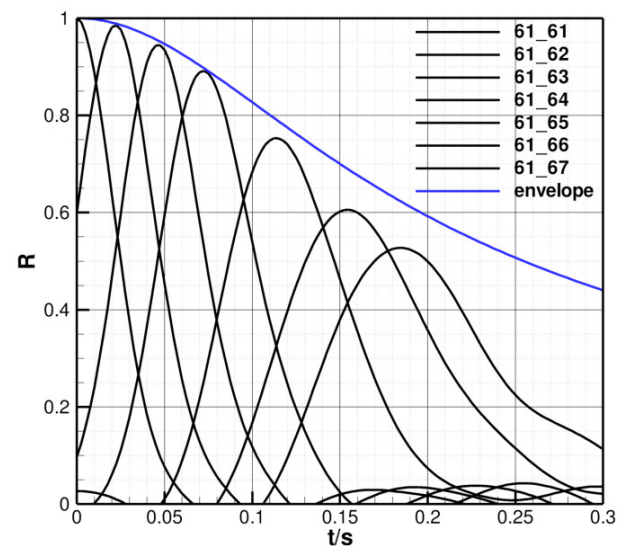

(b)

Figure 7. Spanwise correlation coefficients for unswept airfoil fitted with Gaussian (a); Shear decorrelation in stream wise direction; envelope shows the theoretical decrease (b)

Using Taylor's hypothesis $\left(x / c=\tau \cdot u_{c}\right)$ the autocorrelation can be transformed to a spatial correlation which has again a length scale of 0.0039 .

The statistics for the swept wing show the same behaviour. The spanwise length scale is as before 0.0039 as well as the streamwise length scale shown in Fig. 8. The shear decorrelation on the stream trace (Fig. 8b) fits the theoretical decrease well. The envelope was derived for a straight shear layer; therefore the discrepancy at bigger distances is anticipated.

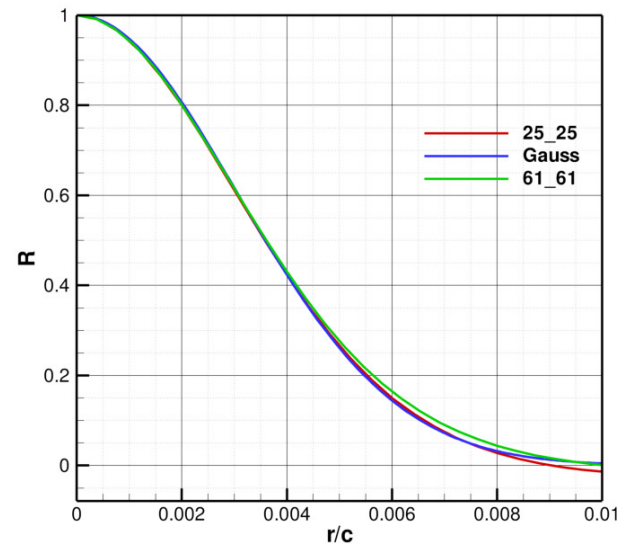

(a)

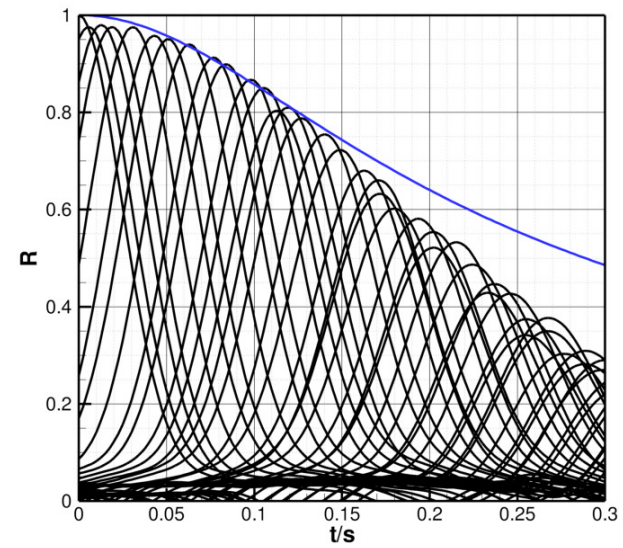

(b)

Figure 8. Spanwise and streamwise correlation coefficients of swept airfoil fitted with Gaussian for the swept wing (a); Shear decorrelation in streamwise direction; envelope shows the theoretical decrease (b)

\section{IV.C. CAA Results}

The RANS results and the stochastic sources prescribed in the previous sections are used to perform the acoustic computations. The spectra shown in this section are always taken at a microphone located at $\Theta=290^{\circ}$, measured in the mathematically positive sense about $\mathrm{z}$ with a value of zero for the positive $\mathrm{x}$ direction. The origin is at $x=y=z=0$ which is nearly the slat trailing edge. The distance to the origin is 1.5 chord length. First of all the spectrum computed with FRPM and PIANO is compared against the spectrum computed with the DG code and FRPM (Fig. 9). Obviously, both codes produce a characteristic slat noise spectrum. There are two peaks around 1 and $2 \mathrm{kHz}$. For frequencies higher than $2 \mathrm{kHz}$ the spectrum exhibits a roll-off. Between 2 and $5 \mathrm{kHz}$ it decreases with a slope proportional to $f^{-2}$ and for frequencies higher than $4 \mathrm{kHz}$ with a slope around $f^{-4}$. The agreement of both spectra is good. Fig. 9(b) 
depicts a 2D FRPM spectrum and a spectrum from Guo's model. ${ }^{10}$ The formula for the slat's spectral shape

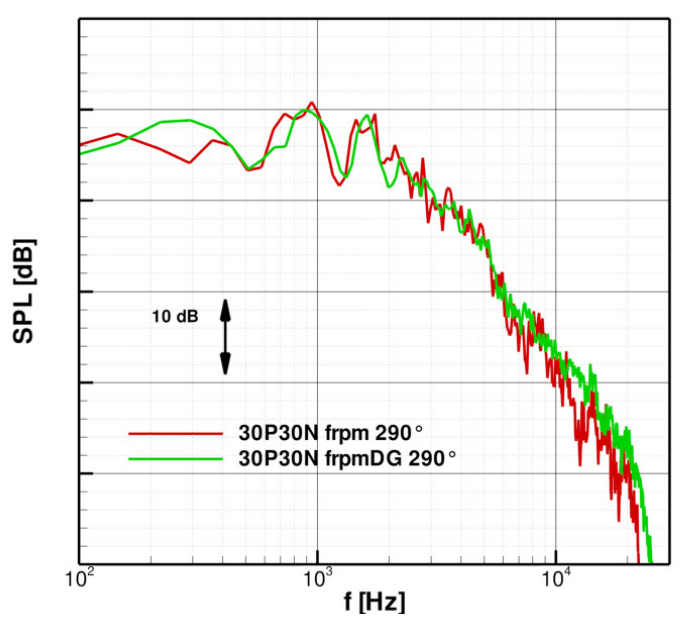

(a)

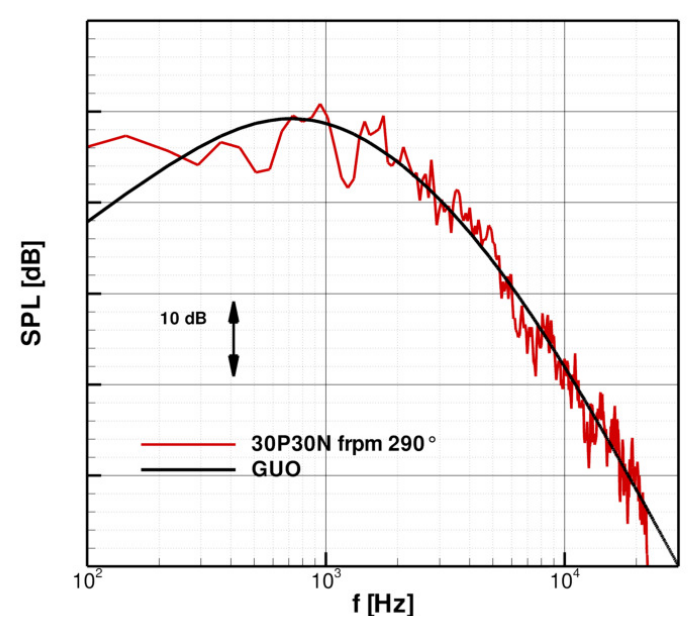

(b)

Figure 9. Spectra compared for the two CAA codes (a); Comparison with Guo's ${ }^{10}$ generic spectrum $\mu_{1}=1.0, \mu_{2}=0.5, \mu_{3}=0.4$, $\mu_{4}=1.0(b)$

function is:

$$
F(f, M)=\frac{M^{2} c_{s}}{c_{0}} \frac{S t^{2}}{\left(1+\mu_{0}^{2} S t^{2}\right)\left(1+\mu_{1}^{2}(1+M)^{2} S t^{2}\right)\left(1+\mu_{2}^{2} M^{2} S t^{2}\right)\left(1+\mu_{3} M S t\right)},
$$

with the slat chord length $c_{s}$, the Mach number $M$, speed of sound $c_{0}$ and the Strouhal number $S t$ built with the slat chord length and the mean flow velocity. The parameters used for Guo's model are $\mu_{1}=1.0$, $\mu_{2}=0.5, \mu_{3}=0.4$ and $\mu_{4}=1.0$. Guo's spectrum fits the computation quite well.

One main difference between $2 \mathrm{D}$ and $3 \mathrm{D}$ is the decay of the effective sound pressure level with the distance. In 3D the effective sound pressure is proportional to the inverse of $r$, whereas in 2D it goes with $r^{-0.5}$. The effective sound pressure is depicted for a line from the origin to the observer point at $\Theta=290^{\circ}$ in Fig. 13). The farfield decay can be observed for 2D and 3D for radii greater than 0.3 chord lengths. That demonstrates that we have a realistic 3D acoustic field in the 3D computation.

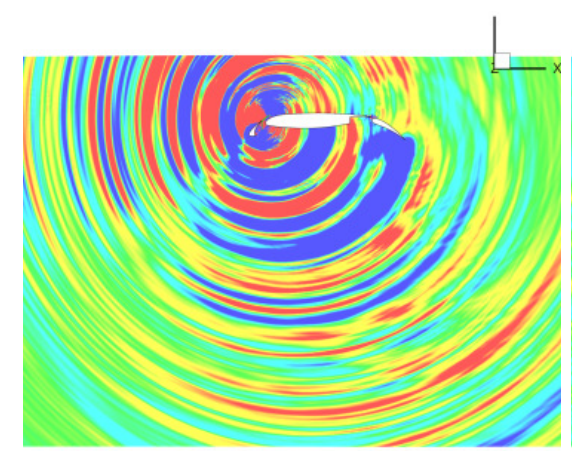

(a) 3D Computation no sweep

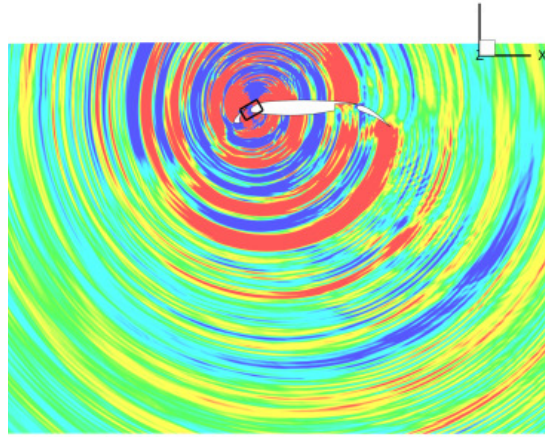

(b) 3D Computation with sweep

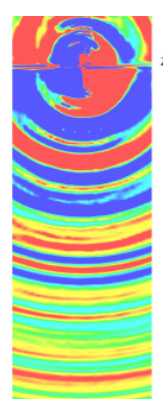

(c) no sweep

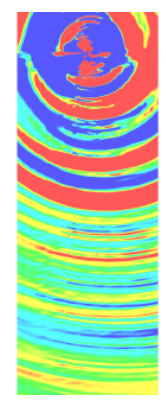

(d) with sweep

Figure 10. Instantaneous pressure perturbations plotted on slices at $z=0$, respectivly $x_{n}=0.025$

Finally the results of the 3D computations for the swept and unswept wing are compared. In Fig. 10 the instantaneous pressure perturbation is plotted for 2 slices; one slice at $x_{n}=0.025$ and one at $z=0.0$. The left picture shows the pressure perturbations for the unswept wing whereas the right one shows it for the swept wing. One can observe the 3D radial propagation of the sound waves emitted from the source region, but for the swept wing case the sound field is convected by the crossflow in z-direction. The Fig. 11 depicts the spectral comparison between 2D FRPM and 3D FRPM for the unswept wing in the left plot and the comparison between the unswept and swept wing on the right. The spectra for the unswept airfoil 
of the 2D and 3D computations show both a characteristic slat noise spectrum. The lower frequencies are not as precise as the higher frequencies because of a relatively short data time interval. Two peaks can be observed at $1 \mathrm{kHz}$ and $1.6 \mathrm{kHz}$. For higher frequencies the spectra decrease. The comparison between

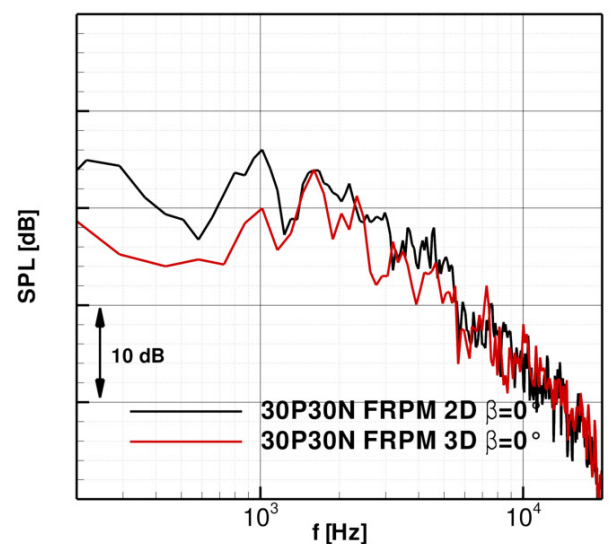

(a) Comparison $2 \mathrm{D} / 3 \mathrm{D} \beta=0^{\circ}$

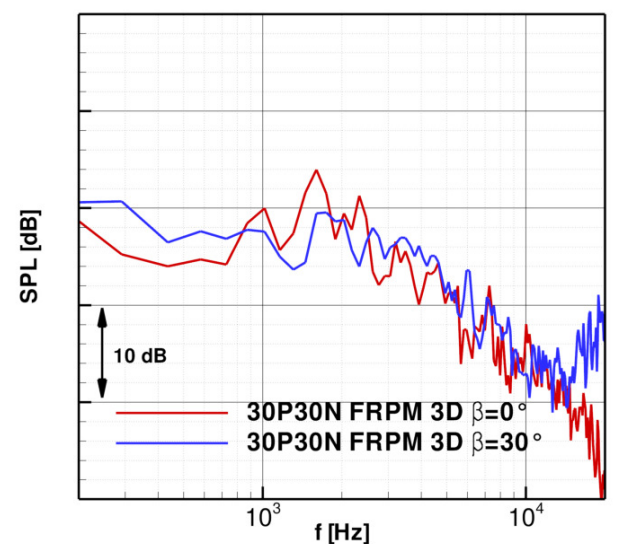

(b) Comparison $3 \mathrm{D} \beta=0^{\circ} / \beta=30^{\circ}$

Figure 11. Spectra at microphone position $r=1.5 \mathrm{c}$ at $\Theta=290^{\circ}$

the swept and unswept wing show a similar behaviour, but for the swept airfoil no spectral peaks can be observed. The spectral levels for the unswept and swept airfoil are similar. This result is expected because the freestream flow speed normal to the leading edge was kept constan. Hence, at the same flight speed a slat noise reduction may be expected for the aifoil with sweep. For frequencies higher than $15 k \mathrm{~Hz}$ a deviation becomes visible in the 3D spectrum, which levels out on a constant level. The reason is the coarseness of the CAA mesh in span-wise direction. The cross flow seems to trigger high frequency disturbances.

The proper simulation of the spanwise coherence is an important factor to accomplish a proper predicted $3 \mathrm{D}$ spectrum. For $2 \mathrm{D}$ predictions the spanwise coherence has to be explicitly taken into account by a $2 \mathrm{D}$ to $3 \mathrm{D}$ correction step to approximately predict a $3 \mathrm{D}$ spectrum. The $2 \mathrm{D}$ to $3 \mathrm{D}$ procedure introduced in Ref. ${ }^{20}$ reveals that the shape for $2 \mathrm{D}$ and $3 \mathrm{D}$ spectra is maintained (i.e. the $2 \mathrm{D}$ to $3 \mathrm{D}$ correction is not frequency dependent). It was to show, that a 3D simulation covers the correct spanwise coherence. If a 3D simulation predicts a similar spectrum as a $2 \mathrm{D}$ simulation with $2 \mathrm{D}$ to $3 \mathrm{D}$ correction implicitly proof is given, that the spanwise coherence is correctly described by the $3 \mathrm{D}$ simulation.

\section{IV.D. Computational time}

The computational time for the 2D and 3D FRPM computations is listed in Tab. 2. The history time identifies the recorded time interval. NoCPUs gives the numbers of CPUs used for the computation. One CPU is always used for FRPM process; the others for the CAA process. The cluster is built out of Intel Xeon CPUs with 2.93GHz. The 3D computations are the first of this kind and therefore the mesh was not

\begin{tabular}{|c|c|c|c|c|c|}
\hline Kind & Interval & noCPUs & CPUtime/CPU & no. of points & Dimensions \\
\hline \hline $2 \mathrm{D}$ & $0.053 s$ & 8 & 0.5 days & $370 K$ & $4.5 c \times 3.0 c$ \\
$3 \mathrm{D}$ & $0.043 s$ & 50 & 11.9 days & $30 M$ & $4.5 c \times 3.0 c \times 1.0 c$ \\
\hline
\end{tabular}

Table 2. Run information

optimal for parallelization. An improved topology should half the computing time.

\section{Conclusion}

It has been shown, that RPM and FRPM produce good results which also fit the theoretical spectra from Guo. The 3D computation has the same spectral characteristics as the $2 \mathrm{D}$ computations in a wide frequency range. Therefore it may be concluded that the coherence length scale assumption for $2 \mathrm{D}$ is correct and that FRPM is able to deal with 3D problems. The high frequency disturbances for the swept wing seem to be 
triggered by a spanwise resolution problem. Therefore it is necessary to spend more points in the source region.
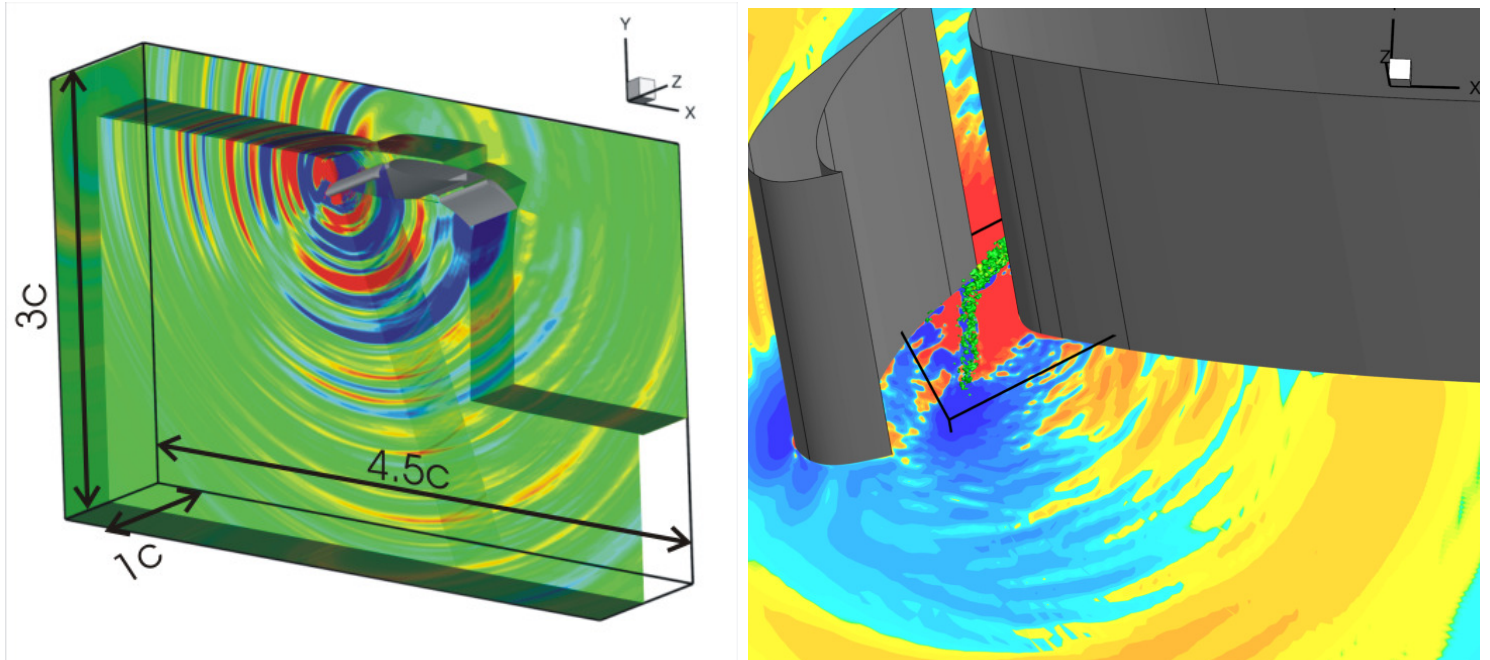

Figure 12. Instantaneous pressure perturbation for 3D FRPM computation (left); View of the source area (right)

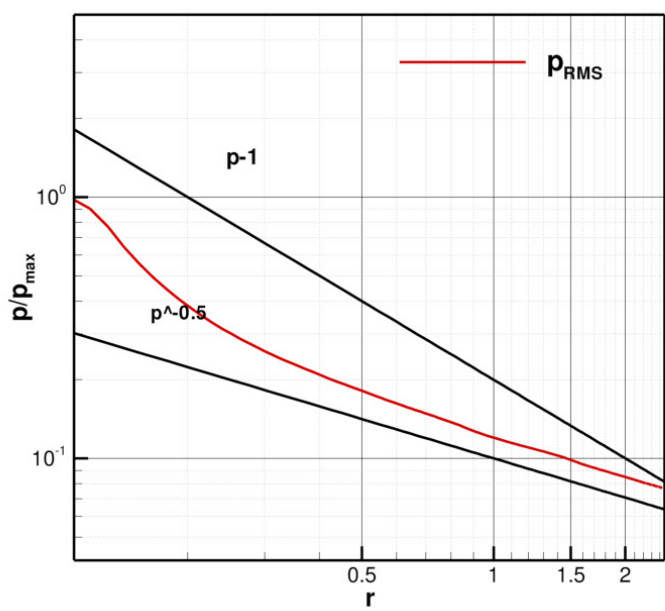

(a) 2D Computation

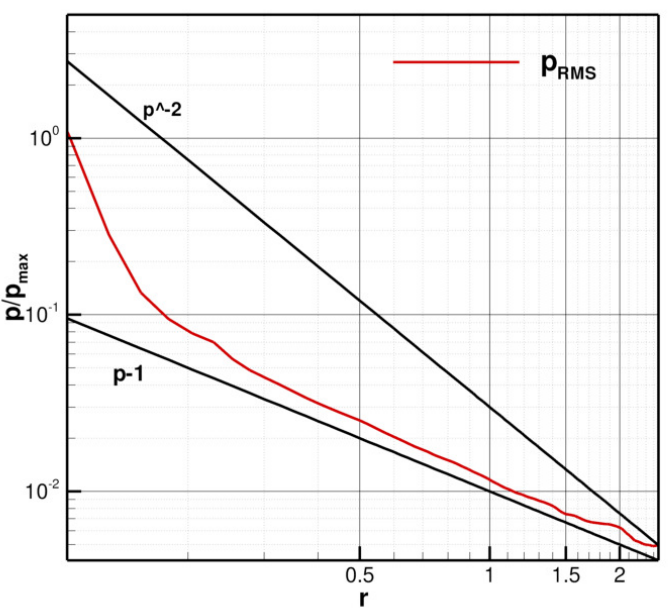

(b) 3D Computation

Figure 13. Overall sound pressure plotted over the distance $r$ on a line radial from the center to $290^{\circ}$

\section{Acknowledgments}

This work was done in a framework of the AERONEXT project in collaboration with Airbus Deutschland. The computational time is provided by Center for Computer Applications in AeroSpace Science and Engineering $\left(\mathrm{C}^{2} \mathrm{~A}^{2} \mathrm{~S}^{2} \mathrm{E}\right)$.

\section{References}

${ }^{1}$ Ewert, R., "RPM-the fast random particle-mesh method to realize unsteady turbulent sound sources and velocity fields for CAA applications," AIAA Paper 2007-3506, 2007.

${ }^{2}$ R. Ewert, J. Dierke, M. P.-P. C. A. R. E. M. S., "CAA-RPM prediction and validation of slat setting influence on broadband high-lift noise generation," AIAA Paper 2010-3833, 2010.

${ }^{3}$ Agarwal, A. and Morris, P. J., "Prediction Method for Broadband Noise from Unsteady Flow in a Slat Cove," AIAA Journal, Vol. 44, 2006. 

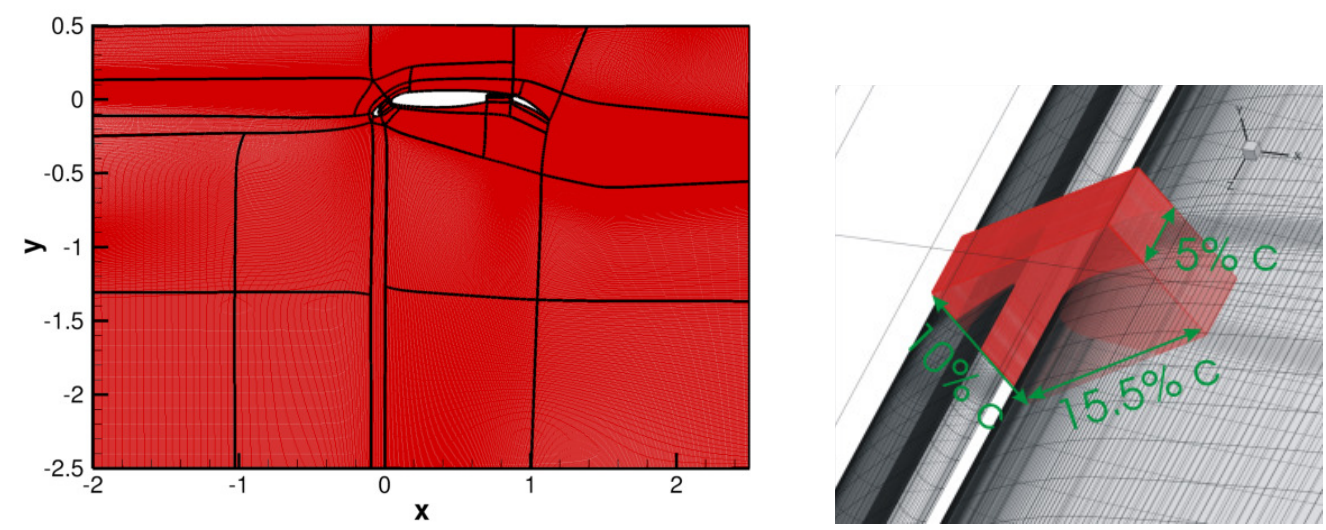

Figure 14. CAA mesh topology (left); Dimensions of the source reagion for $3 D$ with wing chord $c$ (right)

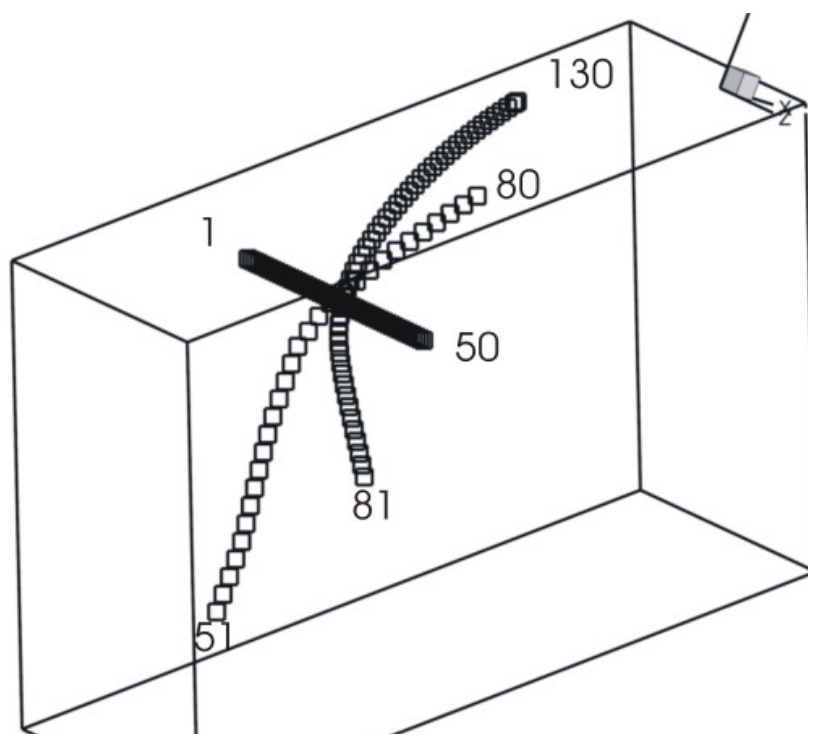

Figure 15. Sensor positions at the source region; 1-50 spanwise near slat trailing edge, 51-80 stream-wise unswept, 81-130 stream-wise swept

${ }^{4}$ Tam, C. and Auriault, L., "Jet Mixing Noise from Fine-Scale Turbulence," AIAA Journal, Vol. 37(2), 1999, pp. $145-153$.

${ }^{5}$ König, D., Schröder, W., and Meinke, M., "Large-eddy simulation of the flow around a high-lift airfoil configuration." Symposium on Turbulence and Shear Flow Phenomena, 2007. 2008.

${ }^{6} \mathrm{Ma}$, Z. and Zhang, X., "Broadband Slat Noise Attenuation with Acoustic Liner Treatment," AIAA Pap. 2008-2964,

${ }^{7}$ Imamura, T., Enomoto, S., Yokokawa, Y., and Yamamoto, K., "Three-Dimensional Unsteady Flow Computations Around a Conventional Slat of High-Lift Devices," AIAA Journal, Vol. 46(5), 2008, pp. 1045-1053.

${ }^{8}$ Lockard, D. P. and Choudhari, M. M., "Noise Radiation from a Leading-Edge Slat," AIAA Pap. 2009-3101, 2009.

${ }^{9}$ Lockard, D. P. and Choudhari, M. M., "The Effect of Cross Flow on Slat Noise," AIAA Pap. 2010-3835, 2010.

${ }^{10}$ Yueping, G., "Aircraft Slat Noise Modelling and Prediction," AIAA Paper 2010-3837, 2010.

${ }^{11}$ Ewert, R., Appel, C., Dierke, J., and Herr, M., "RANS/CAA based predictions of NACA0012 broadband trailing edge noise and experimental valisation," AIAA Pap. 2009-3269, 2009.

${ }^{12}$ Delfs, J. W., Bauer, M., Ewert, R., Grogger, H. A., Lummer, M., and Lauke, T. G. W., "Numerical Simulations of Aerodynamic Noise with DLR's aeroacoustic code PIANO," PIANO, Vol. handbook version 5.2, 2008.

${ }^{13} \mathrm{Tam}$, C. and Webb, J., "Dispersion-Relation-Preserving Finite Difference Schemes for Computational Acoustics," J. Comp. Phys., Vol. 107, 1993, pp. 262-281.

${ }^{14}$ Hu, F. Q., Hussaini, M. Y., and Manthey, J. L., "Low-dissipation and Low-Dispersion Runge-Kutta Schemes for Computational Acoustics," J. Comp. Phys., Vol. 124, 1996, pp. 177-191.

${ }^{15}$ Ewert, R. and Edmunds, R., "CAA slat noise studies applying stochastic sound sources based on solenoidal digital filters," AIAA 2005-2862, Monterey, California, 2005. 


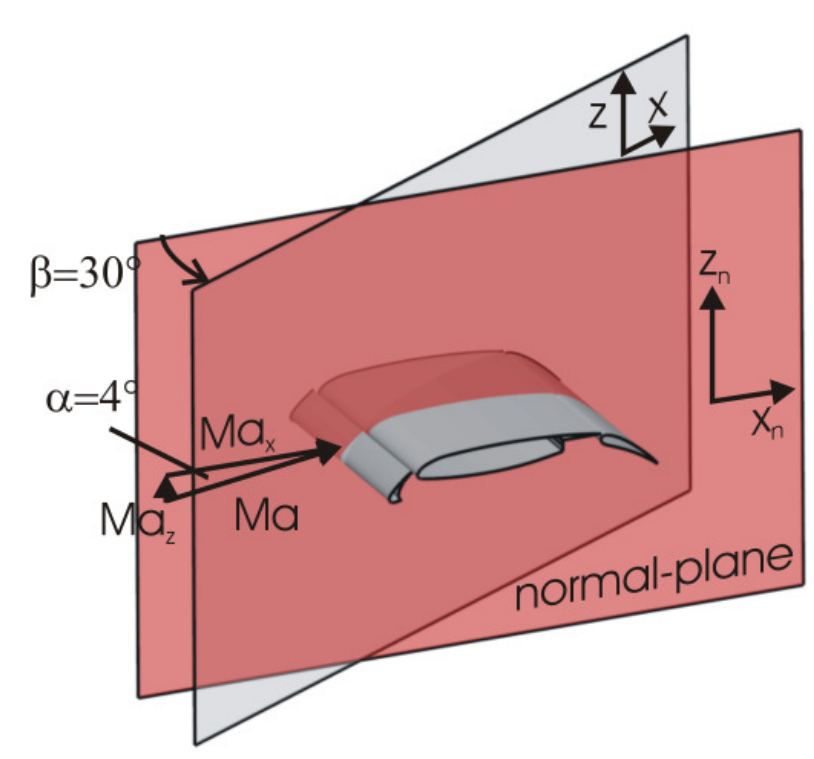

Figure 16. Sketch of the coordinate systems; $n$ indicates the plane with the slat trailing edge as normal

${ }^{16}$ Ewert, R., "Slat noise trend predictions using CAA with stochastic sources from a random particle mesh method (RPM)," AIAA 2006-2667, Cambridge, Massacusetts, 2006.

${ }^{17}$ Ewert, R., "Broadband slat noise prediction based on CAA and stochasic sound sources from a fast random particle-mesh (RPM) method," Computers \& Fluids, Vol. 37, 2008, pp. 369-387.

${ }^{18}$ Ewert, R., "RPM - the fast Random Particle-Mesh method to realize unsteady turbulent sound sources and velocity fields for CAA applications," AIAA 2007-3506, Rome, Italy, 2007.

${ }^{19}$ Siefert, M. and Ewert, R., "Sweeping Sound Generation in Jets Realized with a Random Particle-Mesh Method," AIAA Pap. 2009-3369, 2009.

${ }^{20}$ Ewert, R., Dierke, J., Appel, C., and Herr, M., "RANS/CAA based prediction of NACA 0012 broadband trailing edge noise and experimental validation," AIAA Pap. 2009-3269, 2009. 